\title{
Heterogeneous expectations and speculative behavior in a dynamic multi-asset framework
}

\author{
Carl Chiarella (carl.chiarella@uts.edu.au) \\ School of Finance and Economics \\ University of Technology, Sydney, Australia \\ Roberto Dieci ${ }^{1}$ (rdieci@rimini.unibo.it) \\ Dipartimento di Matematica \\ per le Scienze Economiche e Sociali \\ Università degli Studi di Bologna, Italy \\ Xue-Zhong He (tony.he1@uts.edu.au) \\ School of Finance and Economics \\ University of Technology, Sydney, Australia
}

\begin{abstract}
Following the framework of a one risky - one riskless asset model developed by Brock and Hommes (1998), this paper considers a discrete-time model of a financial market where heterogeneous groups of agents allocate their wealth amongst multiple risky assets and a riskless asset. Agents follow different expectation formation schemes for both first and second moments of the distribution of returns. Instead of using a Walrasian auctioneer scenario as the market clearing mechanism, a market maker scenario is used. In particular, the paper focuses on the case of two risky assets and two agent types, fundamentalists and trend chasers. Conditions for the stability of the "fundamental" equilibrium are established in terms of the key parameters, in particular the extrapolation rate of the trend chasers and the weight of the two groups in the market. Numerical explorations are performed in order to analyze the combined effect of the interaction between heterogeneous traders and the diversification among multiple risky assets. Particular attention is paid to the effect of the correlation between the risky assets. It turns out that investors' anticipated correlation and portfolio diversification do not always have a stabilizing role, but rather may act as a further source of complexity in the financial market.
\end{abstract}

JEL classification: C61; D84; G11; G12

Keywords: Heterogeneous beliefs; Asset pricing; Portfolio choice; Bifurcation analysis; Comovements in stock prices

\footnotetext{
${ }^{1}$ Corresponding author. Address: Universita' degli Studi di Bologna, Facolta' di Economia del Polo di Rimini, Via D. Anghera' 22, I-47900 Rimini (Italy). Phone +39 (0541) 434143; e-mail: rdieci@rimini.unibo.it
} 


\section{Introduction}

In recent years interest has grown in models of asset price dynamics based on the interaction of heterogeneous agents, who seek to learn about the distribution of future returns using different information sets. We cite in particular the models that have been proposed by Day and Huang (1990), Brock and Hommes (1998), Lux (1998), Gaunersdorfer (2000), Chiarella and He (2001, 2003), Chiarella et al. (2002), and Fernandez-Rodriguez et al., (2002). These models in general consider a financial market with one risky asset and one risk-free asset and explore the effect of agents' heterogeneous beliefs about expected return and volatility of the risky asset on the dynamics of asset prices. They show how the interaction between the heterogeneity of the agents and the market trading mechanism can, even in the absence of external random events, cause sustained deviations in prices away from their equilibrium (fundamental) values ${ }^{2}$. Of course, the one risky/one risk-free asset model is merely a first step to understanding price dynamics under heterogeneous agent interaction. Within a multiplerisky-asset framework, the way agents form and update their beliefs about the variance/covariance structure also becomes an important factor in the investors' decision process. A number of papers have appeared recently dealing with the multiple risky asset decision problem within the heterogeneous agent paradigm. We cite in particular Böhm and Chiarella (2005), Böhm and Wenzelburger (2005), Wenzelburger (2004) and Westerhoff (2004). These papers establish an overall framework and discuss various aspects of the problem. ${ }^{3}$ The contribution of the current paper relative to these is, first, to make use of a stylized market maker rather than the Walrasian auctioneer to set prices, second, the updating rules of agents' beliefs are made explicit and are more involved, especially with regard to second moment beliefs. A natural question which arises in this context concerns the effect of asset diversification combined with time varying beliefs about the risk-return structure. In particular, it seems important to understand whether investors' anticipated correlation and diversification among multiple risky assets can have a stabilizing role, or if rather they tend to amplify the price fluctuations which arise due to the interaction of heterogeneous agents, and to what extent portfolio diversification causes interdependence between the price dynamics of different risky assets.

A contribution to the issues raised in the previous paragraph can be found in Chiarella et al. (2005) who develop a discrete time model of financial market dynamics that combines the essential elements of the interacting heterogeneous agents paradigm with the classical model of diversification between two risky assets and a risk-free asset. In particular, a financial market with two risky assets and a riskless asset is considered, the time evolution of which is driven by the interaction of two types of agents, fundamentalists and chartists, together with a market maker. The main findings of the model are that co-movements of prices and returns may arise, brought about by trend extrapolation and time varying beliefs about variances and covariance. Furthermore, changes in beliefs about the risk/return structure can cause volatility to spill-over from the market for one risky asset to the other. However, the model developed in Chiarella et al. (2005) is a very stylized one, in that it considers only two risky assets, it does not take into account the market fractions of the two groups, and the fundamental prices of the risky assets are assumed to be exogenously given.

A sufficiently general framework, which can be generalized in the direction of a multi-asset market, is provided by the single-risky asset dynamic framework of Brock and Hommes (1998), further developed by Chiarella and He $(2001,2003)$ and He (2003). This framework is based on a one-period

\footnotetext{
${ }^{2}$ The interaction between the underlying deterministic driving dynamics and simple noise processes has been shown to constitute one possible source of the fat tails and volatility clustering that are a key feature of asset returns in financial markets. See e.g. Hommes (2001)

${ }^{3}$ In particular, the setup of Böhm and Chiarella (2005) is that of a multi-asset dynamic CAPM with heterogeneous agents - though the dynamic impact of this heterogeneity has not been analyzed in detail - and the questions addressed in that paper are close to those addressed in the present one. In Wenzelburger (2004), this setup has been extended to a model in which myopic agents are allowed to switch between different trading strategies similar to Brock and Hommes (1998).
} 
mean-variance optimization problem of a typical investor, derives a benchmark notion of fundamental price assuming homogeneous expectations, and then perturbs the traditional framework by introducing heterogeneity of beliefs. We follow a similar course in the current paper and derive a general framework for a financial market with multiple risky assets; then we specialize the model to the case of two types of agents, fundamentalists and chartists, who are assumed to have time varying beliefs about the first and the second moments of the distribution of returns. In particular, we highlight the joint effect of diversification and time-varying risk perceptions (via updating of beliefs about the variance/covariance structure) on the amplitude and nature of price fluctuations of the risky assets.

The structure of the paper is as follows. Section 2 develops the general dynamic framework with $n$ risky assets, one risk-free asset and $N$ agent types. In particular Section 2.1 derives the optimal demand of agents for the risky assets in a general setting, as a function of agents' beliefs about future returns, under the assumption of myopic expected utility maximization with CARA utility of wealth function. Sections 2.2 and 2.3 describe how fundamentalists and chartists form and revise their beliefs about expected returns and the variance/covariance structure of returns, while Section 2.4 describes how demands are aggregated by a market maker via a price adjustment rule in the market for each asset and presents the resulting dynamical system for the dynamic evolution of prices and agents' beliefs about asset returns. Section 3 is devoted to the dynamic behavior of the model. In particular, it characterizes the (unique) steady state of the model (section 3.1), discusses its local stability conditions (section 3.2), and performs numerical explorations aimed at capturing the effect of the correlation structure on price fluctuations (section 3.3). Section 4 concludes and indicates possible future research directions. An Appendix containing mathematical details is available on the JEBO Web Site.

\section{The Model}

Following the asset pricing dynamic framework of a financial market with one risk-free asset and one risky asset developed by Brock and Hommes (1998), Chiarella and He (2001, 2003) and He (2003), as well as two risky assets studied in Chiarella et al. (2005) under heterogeneous beliefs, we consider a general asset pricing model with one risk-free asset and multiple risky assets when agents' beliefs are heterogeneous. The asset demands are derived from the standard one-period CARA utility maximization. The major difference is that we assume that agents are boundedly rational by allowing agents to have heterogeneous beliefs about the distribution (in terms of the mean and (co)-variance) of future prices and to update their beliefs dynamically according to certain learning processes based on observed prices, though for simplicity a switching between beliefs is not considered here. ${ }^{4}$

We consider a financial market with $n$ risky assets, indexed by $i=1,2, \ldots, n$, and a risk-free asset, and $N$ types of traders, denoted with the superscript $h=1,2, \ldots, N$. For the risky asset $i(i=1,2, \ldots n)$, we denote by $p_{i, t}$ the price at time $t$, and by $y_{i, t+1}$ the dividend in the trading period $(t, t+1)$. We also denote by $r$ the annual risk-free rate, by $K$ the trading frequency so that $R:=(1+r / K)$ represents the gross risk-free return in the trading period (of length $1 / K$ ). The quantity $R_{i, t+1}:=p_{i, t+1}+y_{i, t+1}-R p_{t}$ represents the excess return per share in $(t, t+1)$. Using $n$-dimensional column vector notation, the above quantities correspond to $\mathbf{p}_{t}$ (prices), $\mathbf{y}_{t+1}$ (dividends) and $\mathbf{R}_{t+1}:=\left(\mathbf{p}_{t+1}+\mathbf{y}_{t+1}-R \mathbf{p}_{t}\right.$ ) (excess returns).

Denote by $W_{t}^{h}$ the wealth of agent-type $h$ at time $t$, by $z_{i, t}^{h}$ the number of shares of risky asset $i$ held by agent $h$ in $(t, t+1)$, and by $E_{t}^{h}, V_{t}^{h}, \operatorname{Cov}_{t}^{h}$ the "beliefs" of agent $h$, conditional upon information at time $t$, about expectation, variance and covariance respectively. The "portfolio" of agent $h$ in $(t, t+1)$

\footnotetext{
${ }^{4}$ A switching mechanism between beliefs could be easily introduced, though it would increase further the dimension of the resulting dynamical system. In addition both the local and global dynamic behavior may change.
} 
is expressed by the vector $\mathbf{z}_{t}^{h}=\left(z_{1, t}^{h}, z_{2, t}^{h}, \cdots, z_{n, t}^{h}\right)^{\top}$, where $\top$ stands for the transpose. Given the portfolio of risky assets and the risk-free asset, the wealth of type- $h$ agent evolves according to

$$
W_{t+1}^{h}=R W_{t}^{h}+\mathbf{z}_{t}^{h \top} \mathbf{R}_{t+1}=R W_{t}^{h}+\mathbf{z}_{t}^{h \top}\left(\mathbf{p}_{t+1}+\mathbf{y}_{t+1}-R \mathbf{p}_{t}\right) .
$$

Accordingly, the conditional expectation and variances/covariances are given by

$$
E_{t}^{h}\left(W_{t+1}^{h}\right)=R W_{t}^{h}+\mathbf{z}_{t}^{h \top} \mathbf{m}_{t}^{h}, \quad V_{t}^{h}\left(W_{t+1}^{h}\right)=\mathbf{z}_{t}^{h \top} \mathbf{V}_{t}^{h} \mathbf{z}_{t}^{h},
$$

where $\mathbf{m}_{t}^{h}:=E_{t}^{h}\left(\mathbf{R}_{t+1}\right)$ is the vector of expected excess returns and $\mathbf{V}_{t}^{h}:=\left[\operatorname{Cov}_{t}^{h}\left(R_{i, t+1}, R_{j, t+1}\right)\right]$, $i, j=1,2, \ldots n$, denotes type- $h$ agent's conditional variance/covariance matrix (assumed to be positive definite) of the excess returns (per share).

\subsection{Asset Demand}

Assume that type- $h$ agent has utility function $u^{h}(W)=-\exp \left(-a^{h} W\right)$, where $a^{h}$ represents the constant absolute risk aversion (CARA) coefficient. Assume the portfolio $\mathbf{z}_{t}^{h}$ of type- $h$ agent is selected to maximize the expected utility of wealth at time $t+1$,

$$
\max _{\mathbf{z}_{t}^{h}} E_{t}^{h}\left[-\exp \left(-a^{h} W_{t+1}^{h}\right)\right] .
$$

As is well known, under the assumption of conditional normality of returns, this is equivalent to solving

$$
\max _{\mathbf{z}_{t}^{h}}\left\{E_{t}^{h}\left(W_{t+1}^{h}\right)-\frac{a^{h}}{2} V_{t}^{h}\left(W_{t+1}^{h}\right)\right\},
$$

which may be expressed as

$$
\max _{\mathbf{z}_{t}^{h}}\left\{R W_{t}^{h}+\mathbf{z}_{t}^{h \top} \mathbf{m}_{t}^{h}-\frac{a^{h}}{2} \mathbf{z}_{t}^{h \top} \mathbf{V}_{t}^{h} \mathbf{z}_{t}^{h}\right\} .
$$

It follows from the first order conditions of the foregoing optimisation problem that the demand functions (in terms of the optimal number of shares) for the risky assets are given by

$$
\mathbf{z}_{t}^{h}=\frac{1}{a^{h}}\left(\mathbf{V}_{t}^{h}\right)^{-1} \mathbf{m}_{t}^{h} .
$$

Equation (1) indicates that demand functions will be different across agents according to their "beliefs". We consider two types of agents, fundamentalists, who have some information on the fundamental values of the risky asset and who believe that prices will return to their fundamental values in the future, and trend chasers or chartists, who may have no information on the fundamental values of the risky asset and who extrapolate the past trends of the historical prices into future price movements. These two types of agents are the most common and popular ones in the literature on heterogeneous agent based models. 


\section{$2.2 \quad$ Fundamentalists}

We assume that the fundamentalists have some (private) information or estimate on the fundamental values, say $\mathbf{p}_{t}^{*}$, of the risky assets and they expect the future prices of the risky assets to evolve according to

$$
E_{t}^{f}\left[\mathbf{p}_{t+1}\right]=\mathbf{p}_{t}^{*}+\alpha\left(\mathbf{p}_{t}-\mathbf{p}_{t}^{*}\right), \quad(0 \leq \alpha \leq 1)
$$

where $(1-\alpha)$ represents the expected speed of adjustment of the prices towards their fundamental values. The fundamentalists are not completely rational in the sense that they do not have perfect foresight on the future price. In reality the fundamentalists would base their estimate of the fundamentals on a detailed analysis of dividends and earnings forecasts, the outlook for key macroeconomic and political factors, and so forth. However to keep the analysis simple and to focus purely on the impact of speculative behavior in a multi-asset framework we assume that the fundamentalists merely base their estimate of the fundamentals on the expected dividend stream, as is assumed in a great deal of the asset-pricing literature. Brock and Hommes (1998) adopt a similar approach. Here we assume in particular that the fundamental prices are those that would prevail in the economy with homogeneous beliefs about the first and second moments of the cum-dividend price processes, and which become the steady state price vector of the dynamical system describing the heterogeneous agent interaction. From this perspective the fundamental price vector should be interpreted as a kind of anchor about which the fluctuations, due to the heterogeneous interactions, occur and which is imposed exogenously as part of the beliefs of the fundamentalists. ${ }^{5}$ As stated earlier this assumption allows us to focus the analysis on the dynamics arising purely from speculative behavior. Of course future research needs to consider the impact of endogenously determined fundamentals using some sort of filtering methodology to learn it.

The following Lemma (the proof of which can be found in Appendix A1, available on the JEBO Web Site) states the main assumptions and results about the fundamental price vector.

Lemma. Assume that:-

(H1) Agents are homogeneous in their risk aversion coefficients, and their beliefs with respect to mean and (co)-variance of the excess return $\mathbf{R}_{t+1}$ :

$$
a^{h}:=a, \quad E_{t}^{h}\left(\mathbf{R}_{t+1}\right):=\mathbf{m}_{t}, \quad \mathbf{V}_{t}^{h}=\mathbf{V} \equiv\left[\begin{array}{cccc}
\sigma_{1}^{2} & \rho_{12} \sigma_{1} \sigma_{2} & \cdots & \rho_{1 n} \sigma_{1} \sigma_{n} \\
\rho_{12} \sigma_{1} \sigma_{2} & \sigma_{2}^{2} & \cdots & \rho_{2 n} \sigma_{2} \sigma_{n} \\
\vdots & \vdots & \ddots & \vdots \\
\rho_{1 n} \sigma_{1} \sigma_{n} & \rho_{2 n} \sigma_{2} \sigma_{n} & \cdots & \sigma_{n}^{2}
\end{array}\right],
$$

where $\sigma_{i}$ and $\rho_{i j}, i, j=1,2, \ldots, n$, denote, respectively, the standard deviation of the excess return (per share) of asset $i$ and the correlation between excess returns $i$ and $j$.

(H2) Agents have common beliefs about the dividend process, which is i.i.d. with $E_{t}\left[\mathbf{y}_{t+k}\right]=\overline{\mathbf{y}}$ $(k=1,2, \ldots)$.

(H3) The fundamental solution satisfies the transversality (no-bubbles) conditions

$$
\lim _{k \rightarrow+\infty} E_{t}\left[p_{i, t+k}\right] / R^{k}=0, \quad i=1,2, \ldots, n .
$$

\footnotetext{
${ }^{5}$ Note also the coincidence of the "fundamental solution" derived in the present section with the Böhm and Chiarella (2005) case under homogeneous expectations.
} 
Then the unique fundamental price is given by

$$
\mathbf{p}_{t}=\mathbf{p}^{*}:=\frac{\overline{\mathbf{y}}^{\#}}{R-1}=\frac{1}{R-1}\left(\overline{\mathbf{y}}-a \mathbf{V} \mathbf{z}^{s}\right),
$$

where $\mathbf{z}^{s}:=\left[z_{i}^{s}\right](i=1,2, \ldots n)$ is the supply (column) vector, whose $i$-th element $z_{i}^{s}$ is the total stock of asset $i$ per investor, and $\overline{\mathbf{y}}^{\#}:=\overline{\mathbf{y}}-a \mathbf{V} \mathbf{z}^{s}$ denotes the risk-adjusted dividends.

The above Lemma gives a clear idea of how the constant fundamental price of each asset is affected by the variance/covariance matrix and by the supply of all the other assets.

The fundamentalists are also assumed to have the same constant beliefs about the variance/covariance structure of the excess returns with $\mathbf{V}_{t}^{f}=\mathbf{V}$, that is, for $i, j=1,2, \ldots, n$,

$$
\operatorname{Cov}_{t}^{f}\left[R_{i, t+1}, R_{j, t+1}\right]=\rho_{i j} \sigma_{i} \sigma_{j}, \quad V_{t}^{f}\left[R_{i, t+1}\right]=\sigma_{i}^{2} .
$$

Then

$$
\mathbf{m}_{t}^{f}:=E_{t}^{f}\left[\mathbf{R}_{t+1}\right]=E_{t}^{f}\left[\mathbf{p}_{t+1}+\mathbf{y}_{t+1}-R \mathbf{p}_{t}\right]=(\alpha-R) \mathbf{p}_{t}+(1-\alpha) \mathbf{p}^{*}+\overline{\mathbf{y}} .
$$

In order to obtain a model suitable for eventual calibration to real data, we need to take into account the "trading period" dividend and relate the variance/covariance estimates to the trading frequency. To keep the model mathematically tractable, we assume that fundamentalists ${ }^{6}$ form their beliefs about variances and covariances of excess returns $R_{i, t+1}=p_{i, t+1}+y_{i, t+1}-R p_{i, t}$ in the "trading period", in the following way. Given the annual standard deviation (s.d.) of the price of asset $i$, which we denote by $\widetilde{\sigma}_{i}$, the estimated s.d. of the price of asset $i$ per "trading period" is $\bar{\sigma}_{i}:=\widetilde{\sigma}_{i} / \sqrt{K}$, so that $V_{t}^{f}\left(p_{i, t+1}\right)=\bar{\sigma}_{i}^{2} \equiv \tilde{\sigma}_{i}^{2} / K$. In order to include the effect of the "trading period" dividend, we assume that agents compute the s.d. of the "cum-price" in proportion to the s.d. of the price, which can be expressed in general by assuming ${ }^{7} V_{t}^{f}\left(R_{i, t+1}\right)=q_{i}^{2} V_{t}^{f}\left(p_{i, t+1}\right), \operatorname{Cov}_{t}^{f}\left[R_{i, t+1}, R_{j, t+1}\right]=$ $q_{i} q_{j} \operatorname{Cov}_{t}^{f}\left[p_{i, t+1}, p_{j, t+1}\right]$. Thus

$$
\begin{gathered}
\sigma_{i}^{2}:=V_{t}^{f}\left[R_{i, t+1}\right]=V_{t}^{f}\left[p_{i, t+1}+y_{i, t+1}\right]=q_{i}^{2} \frac{\widetilde{\sigma}_{i}^{2}}{K}=q_{i}^{2} \bar{\sigma}_{i}^{2}, \\
\operatorname{Cov}_{t}^{f}\left[R_{i, t+1}, R_{j, t+1}\right]=q_{i} q_{j} \frac{\rho_{i j} \widetilde{\sigma}_{i} \widetilde{\sigma}_{j}}{K}=q_{i} q_{j} \rho_{i j} \bar{\sigma}_{i} \bar{\sigma}_{j},
\end{gathered}
$$

or with vector notation

$$
\mathbf{V}_{t}^{f} \equiv \mathbf{V}=\mathbf{Q}^{\top} \overline{\mathbf{V}} \mathbf{Q}
$$

where

$$
\overline{\mathbf{V}}=\frac{1}{K}\left[\begin{array}{cccc}
\tilde{\sigma}_{1}^{2} & \rho_{12} \widetilde{\sigma}_{1} \widetilde{\sigma}_{2} & \cdots & \rho_{1 n} \widetilde{\sigma}_{1} \widetilde{\sigma}_{n} \\
\rho_{12} \widetilde{\sigma}_{1} \widetilde{\sigma}_{2} & \widetilde{\sigma}_{2}^{2} & \cdots & \rho_{2 n} \widetilde{\sigma}_{2} \widetilde{\sigma}_{n} \\
\vdots & \vdots & \ddots & \vdots \\
\rho_{1 n} \widetilde{\sigma}_{1} \widetilde{\sigma}_{n} & \rho_{2 n} \tilde{\sigma}_{2} \widetilde{\sigma}_{n} & \cdots & \widetilde{\sigma}_{n}^{2}
\end{array}\right]
$$

and $\mathbf{Q} \equiv \operatorname{diag}\left(q_{1}, q_{2}, \ldots, q_{n}\right)=\mathbf{Q}^{\top}$.

\footnotetext{
${ }^{6}$ A similar assumption will be made for chartists

${ }^{7}$ In particular, for calibration purposes agents would set $q_{i}=1+g_{i} / K,(i=1,2, \ldots, n)$ where $g_{i}$ represents an estimate of the average (per annual) dividend yield of asset $i$, or alternatively $q_{i}=R=1+r / K$. For real daily data $r / K$ is of the order of $10^{-4}$ so in our numerical examples we will assume $q_{i}=1$ for simplicity.
} 


\subsection{Chartists}

We assume that the chartists' expected prices are computed according to

$$
E_{t}^{c}\left[\mathbf{p}_{t+1}\right]=\mathbf{p}_{t}+\beta\left(\mathbf{p}_{t}-\mathbf{u}_{t}\right),
$$

where $\beta \geq 0$ is the price extrapolation parameter (for simplicity assumed constant across all assets) and $\mathbf{u}_{t}=\left[u_{i, t}\right], i=1,2, \ldots, n$, is a (column) vector of sample means. Then, under assumption (H2),

$$
\mathbf{m}_{t}^{c}:=E_{t}^{c}\left[\mathbf{R}_{t+1}\right]=E_{t}^{c}\left[\mathbf{p}_{t+1}+\mathbf{y}_{t+1}-R \mathbf{p}_{t}\right]=(1+\beta-R) \mathbf{p}_{t}-\beta \mathbf{u}_{t}+\overline{\mathbf{y}}
$$

The beliefs of the chartists about the variance/covariance matrix of prices in the trading period (denoted by $\mathbf{B}_{t}$ ) are assumed to consist of a constant component and a time varying component and so may be written as

$$
\mathbf{B}_{t}=\overline{\mathbf{V}}+\Xi_{t}
$$

Here we assume for simplicity that the constant component is equal to the fundamental component given by (7). Furthermore the time varying component is assumed to be updated in each period as a function of deviations of prices from sample means. The matrix

$$
\Xi_{t}:=\left[\begin{array}{cccc}
\gamma_{11} v_{1, t} & \gamma_{12} k_{12, t} & \cdots & \gamma_{1 n} k_{1 n, t} \\
\gamma_{12} k_{12, t} & \gamma_{22} v_{2, t} & \cdots & \gamma_{2 n} k_{2 n, t} \\
\vdots & \vdots & \ddots & \vdots \\
\gamma_{1 n} k_{1 n, t} & \gamma_{2 n} k_{2 n, t} & \cdots & \gamma_{n n} v_{n, t}
\end{array}\right]
$$

contains the time-varying components which are assumed proportional, through the sensitivity coefficients $\gamma_{i j} \geq 0$, to the sample variances/covariances based on historical volatility and correlation. We also denote by $\boldsymbol{\Sigma}_{t}$ the matrix of such time-varying sample variances/covariances, so that ${ }^{8}$

$$
\boldsymbol{\Sigma}_{t}:=\left[\begin{array}{cccc}
v_{1, t} & k_{12, t} & \cdots & k_{1 n, t} \\
k_{12, t} & v_{2, t} & \cdots & k_{2 n, t} \\
\vdots & \vdots & \ddots & \vdots \\
k_{1 n, t} & k_{2 n, t} & \cdots & v_{n, t}
\end{array}\right]
$$

Similar to He (2003) and Chiarella et al. (2005), the learning processes about the sample means, variances and covariances $\left(\mathbf{u}_{t}\right.$ and $\left.\boldsymbol{\Sigma}_{t}\right)$ are assumed to follow

$$
\begin{gathered}
\mathbf{u}_{t}=\delta \mathbf{u}_{t-1}+(1-\delta) \mathbf{p}_{t} \\
\boldsymbol{\Sigma}_{t}=\delta \boldsymbol{\Sigma}_{t-1}+\delta(1-\delta)\left(\mathbf{p}_{t}-\mathbf{u}_{t-1}\right)\left(\mathbf{p}_{t}-\mathbf{u}_{t-1}\right)^{\top},
\end{gathered}
$$

where $\delta, 0<\delta<1$, represents a "memory" parameter, in the sense that the higher is $\delta$, the higher is the weight given to past prices in the computation of the sample means, variances and covariances. Notice that the higher is $\delta$, the stronger is the effect of chartist extrapolation. In fact, the processes (10) and (11) can be considered as limiting cases of geometric decay processes when the memory lag length tends to infinity, that is, for $i, j=1,2, \ldots n$, they are equivalent to

$$
u_{i, t}=\sum_{s=0}^{\infty}(1-\delta) \delta^{s} p_{i, t-s}
$$

\footnotetext{
${ }^{8}$ In some of the numerical experiments performed in Section 3.3 we will assume $\gamma_{i j}=\gamma(i, j=1,2, \ldots, n)$ which implies $\Xi_{t}=\gamma \Sigma_{t}$.
} 


$$
\begin{gathered}
v_{i, t}=\sum_{s=0}^{\infty}(1-\delta) \delta^{s}\left(p_{i, t-s}-u_{i, t}\right)^{2}, \\
k_{i j, t}=\sum_{s=0}^{\infty}(1-\delta) \delta^{s}\left(p_{i, t-s}-u_{i, t}\right)\left(p_{j, t-s}-u_{j, t}\right) .
\end{gathered}
$$

Using the same scaling matrix $\mathbf{Q}$ as the fundamentalists (since knowledge about the dividend process is assumed to be common), the chartists then compute the conditional variances/covariances of the excess returns (per share) according to

$$
\mathbf{V}_{t}^{c}=\mathbf{Q}^{\top}\left(\overline{\mathbf{V}}+\Xi_{t}\right) \mathbf{Q}
$$

\subsection{The asset prices under a market maker scenario}

We now specify the price setting mechanism for each asset. We assume that in each period, a market maker ${ }^{9}$ clears the market, by taking an offsetting long or short position, and adjusts the price of each risky asset as a function of the excess demand. ${ }^{10}$ This is expressed by the price setting equations

$$
\mathbf{p}_{t+1}=\mathbf{p}_{t}+\mu\left(n^{f} \mathbf{z}_{t}^{f}+n^{c} \mathbf{z}_{t}^{c}-\mathbf{z}^{s}\right)
$$

where $\mu>0$ is the market maker's price adjustment parameter, $n^{f}$ and $n^{c}\left(=1-n^{f}\right)$ represent the fractions of fundamentalists and chartists, respectively, so that the quantity $\left(n^{f} \mathbf{z}_{t}^{f}+n^{c} \mathbf{z}_{t}^{c}-\mathbf{z}^{s}\right)$ represents the average excess demand per investor. The following Proposition summarizes the asset price dynamics under heterogeneous beliefs, assuming that agents are homogeneous in their risk aversion coefficients.

Proposition 1. Taking account of the different beliefs (5), (6), (8) and (12), of fundamentalists and chartists, the model results in the following nonlinear discrete-time dynamical system

$$
\left\{\begin{array}{l}
\mathbf{p}_{t+1}=\mathbf{p}_{t}+\mu\left[n^{f} \mathbf{z}_{t}^{f}+\left(1-n^{f}\right) \mathbf{z}_{t}^{c}-\mathbf{z}^{s}\right] \\
\mathbf{u}_{t+1}=\delta \mathbf{u}_{t}+(1-\delta) \mathbf{p}_{t+1} \\
\boldsymbol{\Sigma}_{t+1}=\delta \boldsymbol{\Sigma}_{t}+\delta(1-\delta)\left(\mathbf{p}_{t+1}-\mathbf{u}_{t}\right)\left(\mathbf{p}_{t+1}-\mathbf{u}_{t}\right)^{\top}
\end{array}\right.
$$

where the fundamentalist and chartist optimal demands,

$$
\mathbf{z}_{t}^{f}=\frac{1}{a}\left(\mathbf{Q}^{\top} \overline{\mathbf{V}} \mathbf{Q}\right)^{-1}\left[(\alpha-R) \mathbf{p}_{t}+(1-\alpha) \mathbf{p}^{*}+\overline{\mathbf{y}}\right]
$$

and

$$
\mathbf{z}_{t}^{c}=\frac{1}{a}\left(\mathbf{Q}^{\top}\left(\overline{\mathbf{V}}+\Xi_{t}\right) \mathbf{Q}\right)^{-1}\left[(1+\beta-R) \mathbf{p}_{t}-\beta \mathbf{u}_{t}+\overline{\mathbf{y}}\right]
$$

\footnotetext{
${ }^{9} \mathrm{~A}$ price setting mechanism based on the market maker scenario has been used by a number of authors, in particular Day and Huang (1990) and Beja and Goldman (1980).

${ }^{10}$ More precisely, at the beginning of the trading period (time $t$ ) the market maker announces the new prices $\mathbf{p}_{t}$ and agents form their beliefs about the excess returns $\mathbf{R}_{t+1}$ as well as their demands $\mathbf{z}_{t}^{f}$ and $\mathbf{z}_{t}^{c}$ (that will result in general in positive or negative excess demand for each asset). The market maker then sets excess demands to zero by changing his/her inventory position, and transactions take place at the prices $\mathbf{p}_{t}$. At the beginning of the next trading period (time $t+1$ ) dividend payments are realized and the market maker announces the new prices $\mathbf{p}_{t+1}$, by adjusting the price of each asset in the direction of the excess demand of the previous period.
} 
are functions of the state variables $\mathbf{p}_{t}, \mathbf{u}_{t}$, and $\boldsymbol{\Sigma}_{t}$ at time $t^{11}$, while the fundamental prices are given by

$$
\mathbf{p}^{*}=\frac{1}{R-1}\left(\overline{\mathbf{y}}-a \mathbf{V} \mathbf{z}^{s}\right)=\frac{1}{R-1}\left[\overline{\mathbf{y}}-a\left(\mathbf{Q}^{\top} \overline{\mathbf{V}} \mathbf{Q}\right) \mathbf{z}^{s}\right]
$$

In order to obtain some insight into the price dynamics of the complete model, ${ }^{12}$ we will focus on the case with two risky assets in the following discussion.

\section{Price Dynamics of the Complete Model}

In this section, we present first a result on the existence of the unique steady-state of the complete model. The local stability of the steady-state is then analyzed. Finally, an analysis of the impact of exogenous and perceived correlations on the dynamics of the prices is conducted by use of numerical simulations.

\subsection{Existence and uniqueness of the steady-state}

When the fundamental price is given by the constant vector $\mathbf{p}^{*}$, it is interesting to know if it is also the unique steady-state of the complete model (13) with heterogeneous agent. This is indeed the case as is confirmed by the following Proposition, which is easily verified.

Proposition 2. The dynamical system (13) has the unique, fundamental steady state, defined by

$$
\mathbf{p}_{t}=\mathbf{u}_{t}=\mathbf{p}^{*}, \quad \boldsymbol{\Sigma}_{t}=\mathbf{0} .
$$

Note that, at the fundamental steady state, $\mathbf{z}_{t}^{f}=\mathbf{z}_{t}^{c}=\mathbf{z}^{s}, \mathbf{V}_{t}^{c}=\mathbf{V}_{t}^{f}=\mathbf{V}=\mathbf{Q}^{\top} \overline{\mathbf{V}} \mathbf{Q}$, and $E_{t}^{c}\left[\mathbf{R}_{t+1}\right]=E_{t}^{f}\left[\mathbf{R}_{t+1}\right]=a \mathbf{V} \mathbf{z}^{s}$.

\subsection{Local stability analysis}

In the case of a single risky asset, $n=1$, the dynamical system (13) becomes

$$
\left\{\begin{array}{l}
p_{t+1}=p_{t}+\mu\left[n^{f} z_{t}^{f}+\left(1-n^{f}\right) z_{t}^{c}-z^{s}\right] \\
u_{t+1}=\delta u_{t}+(1-\delta) p_{t+1} \\
v_{t+1}=\delta v_{t}+\delta(1-\delta)\left(p_{t+1}-u_{t}\right)^{2}
\end{array}\right.
$$

where the agents' demand functions are defined as

$$
z_{t}^{f}=\frac{(\alpha-R) p_{t}+(1-\alpha) p^{*}+\bar{y}}{a q^{2} \widetilde{\sigma}^{2} / K}, \quad z_{t}^{c}=\frac{(1+\beta-R) p_{t}-\beta u_{t}+\bar{y}}{a q^{2}\left(\widetilde{\sigma}^{2} / K+\gamma v_{t}\right)},
$$

\footnotetext{
${ }^{11}$ Notice that the quantities $\mathbf{p}_{t+1}$ and $\left(\mathbf{p}_{t+1}-\mathbf{u}_{t}\right)\left(\mathbf{p}_{t+1}-\mathbf{u}_{t}\right)^{\top}$, which appear in the right-hand side of the second and third equations of (13), are also functions of the state at time $t$.

${ }^{12}$ In the case of $n$ risky assets and a riskless asset, the resulting dynamical system is of dimension $3 n+\left(n^{2}-n\right) / 2$.
} 
and the fundamental value is given by

$$
p^{*}=\frac{\bar{y}-a z^{s} q^{2} \widetilde{\sigma}^{2} / K}{R-1}
$$

A detailed bifurcation analysis of a three-dimensional, single-risky-asset dynamic model very similar to (14), has been performed by He (2003). In particular, for the model analyzed by He (2003), as well as for the system (14), local stability analysis of the steady state and numerical simulation show that the fundamental equilibrium may become unstable, through a Neimark-Hopf bifurcation, for high trend chaser extrapolation rate $(\beta)$, high trend chaser fraction $\left(n^{c}=1-n^{f}\right)$, high price adjustment coefficient $(\mu)$, and high "memory" parameter $(\delta)$. In a similar vein we find in the numerical experiments reported below evidence that the Neimark-Hopf bifurcation is of supercritical type, so that an attracting closed curve is created when the parameters cross the bifurcation boundary. However, in the presence of a high fundamentalist fraction $\left(n^{f}\right)$ or strong fundamentalist reaction (i.e. low $\alpha$ ) the steady state may become unstable through a flip-bifurcation. Moreover, chaotic dynamics are likely to occur under parameter regimes where strong chartist extrapolation is associated with strong fundamentalist reaction.

We henceforth focus our attention on the case of two risky assets, $n=2$. For simplicity, in the following analysis and in the numerical simulations it is assumed that $\gamma_{11}=\gamma_{22}:=\gamma^{13}$ We also set $\gamma_{k}:=\gamma_{12}=\gamma_{21}, k:=k_{12}=k_{21}, \rho:=\rho_{12}=\rho_{21}$. The model (13) then becomes the seven-dimensional dynamical system

$$
\left\{\begin{array}{l}
p_{i, t+1}=p_{i, t}+\mu\left[n^{f} z_{i, t}^{f}+\left(1-n^{f}\right) z_{i, t}^{c}-z_{i}^{s}\right], \quad(i=1,2) \\
u_{i, t+1}=\delta u_{i, t}+(1-\delta) p_{i, t+1}, \quad(i=1,2) \\
v_{i, t+1}=\delta v_{i, t}+\delta(1-\delta)\left(p_{i, t+1}-u_{i, t}\right)^{2}, \quad(i=1,2) \\
k_{t+1}=\delta k_{t}+\delta(1-\delta)\left(p_{1, t+1}-u_{1, t}\right)\left(p_{2, t+1}-u_{2, t}\right),
\end{array}\right.
$$

while the fundamental prices turn out to be given by

$$
\begin{aligned}
& p_{1}^{*}=\frac{\bar{y}_{1}-a\left(z_{1}^{s} q_{1}^{2} \widetilde{\sigma}_{1}^{2} / K+z_{2}^{s} \rho q_{1} q_{2} \widetilde{\sigma}_{1} \widetilde{\sigma}_{2} / K\right)}{R-1}, \\
& p_{2}^{*}=\frac{\bar{y}_{2}-a\left(z_{1}^{s} \rho q_{1} q_{2} \widetilde{\sigma}_{1} \widetilde{\sigma}_{2} / K+z_{2}^{s} q_{2}^{2} \widetilde{\sigma}_{2}^{2} / K\right)}{R-1},
\end{aligned}
$$

and the demand functions can be rewritten as the sum of a direct demand and a hedging demand

$$
\begin{gathered}
z_{1, t}^{f}=\frac{(\alpha-R) p_{1, t}+(1-\alpha) p_{1}^{*}+\bar{y}_{1}}{a q_{1}^{2}\left(1-\rho^{2}\right) \widetilde{\sigma}_{1}^{2} / K}-\frac{\rho\left[(\alpha-R) p_{2, t}+(1-\alpha) p_{2}^{*}+\bar{y}_{2}\right]}{a q_{1} q_{2}\left(1-\rho^{2}\right) \widetilde{\sigma}_{1} \widetilde{\sigma}_{2} / K}, \\
z_{2, t}^{f}=\frac{(\alpha-R) p_{2, t}+(1-\alpha) p_{2}^{*}+\bar{y}_{2}}{a q_{2}^{2}\left(1-\rho^{2}\right) \widetilde{\sigma}_{2}^{2} / K}-\frac{\rho\left[(\alpha-R) p_{1, t}+(1-\alpha) p_{1}^{*}+\bar{y}_{1}\right]}{a q_{1} q_{2}\left(1-\rho^{2}\right) \widetilde{\sigma}_{1} \widetilde{\sigma}_{2} / K}, \\
z_{1, t}^{c}=\frac{(1+\beta-R) p_{1, t}-\beta u_{1, t}+\bar{y}_{1}}{a q_{1}^{2}\left(1-\rho_{t}^{(c) 2}\right)\left(\widetilde{\sigma}_{1}^{2} / K+\gamma v_{1, t}\right)}-\frac{\rho_{t}^{(c)}\left[(1+\beta-R) p_{2, t}-\beta u_{2, t}+\bar{y}_{2}\right]}{a q_{1} q_{2}\left(1-\rho_{t}^{(c) 2}\right) \sqrt{\left(\widetilde{\sigma}_{1}^{2} / K+\gamma v_{1, t}\right)\left(\widetilde{\sigma}_{2}^{2} / K+\gamma v_{2, t}\right)}},
\end{gathered}
$$

\footnotetext{
${ }^{13}$ This assumption has no effect on the local stability conditions, that are independent of the parameters $\gamma_{i j}$. See Appendix A2.
} 


$$
z_{2, t}^{c}=\frac{(1+\beta-R) p_{2, t}-\beta u_{2, t}+\bar{y}_{2}}{a q_{2}^{2}\left(1-\rho_{t}^{(c) 2}\right)\left(\widetilde{\sigma}_{2}^{2} / K+\gamma v_{2, t}\right)}-\frac{\rho_{t}^{(c)}\left[(1+\beta-R) p_{1, t}-\beta u_{1, t}+\bar{y}_{1}\right]}{a q_{1} q_{2}\left(1-\rho_{t}^{(c) 2}\right) \sqrt{\left(\widetilde{\sigma}_{1}^{2} / K+\gamma v_{1, t}\right)\left(\widetilde{\sigma}_{2}^{2} / K+\gamma v_{2, t}\right)}},
$$

where the estimated chartist correlation at time $t$ is given by

$$
\rho_{t}^{(c)}=\frac{\rho \widetilde{\sigma}_{1} \widetilde{\sigma}_{2} / K+\gamma_{k} k_{t}}{\sqrt{\left(\widetilde{\sigma}_{1}^{2} / K+\gamma v_{1, t}\right)\left(\widetilde{\sigma}_{2}^{2} / K+\gamma v_{2, t}\right)}} .
$$

Because of the higher dimension of the system, the local stability of the fundamental steady-state and bifurcation analysis of the portfolio with two risky assets is rather difficult in general. However, when the "exogenous" correlation coefficient of the excess returns $\rho$, which is a commonly held belief among the agents, is zero, the local stability and bifurcation of the dynamic portfolio model with two risky assets is actually determined by that of two portfolios each with a single risky asset. ${ }^{14}$ More precisely, we have the following result the proof of which can be found in Appendix A2 (available on the JEBO Web Site).

Proposition 3. Assume $\rho=0$ and set $\sigma^{2} \equiv \min \left\{\sigma_{1}^{2}, \sigma_{2}^{2}\right\}$. The fundamental steady-state is locally asymptotically stable (LAS) if either

$$
\mu<2 a \sigma^{2} /(R-\alpha), \quad n_{N H}^{f}(\beta)<n^{f} \leq 1,
$$

or

$$
\mu \geq 2 a \sigma^{2} /(R-\alpha), \quad n_{N H}^{f}(\beta)<n^{f}<n_{F}^{f}(\beta)
$$

where

$$
n_{N H}^{f}(\beta):=\frac{1+\beta-R-(1-\delta) a \sigma^{2} /(\delta \mu)}{1+\beta-\alpha}
$$

and

$$
n_{F}^{f}(\beta):=\frac{2 a \sigma^{2}+2 \mu \beta \delta /(1+\delta)-\mu(R-1)}{\mu[(1-\alpha)+2 \beta \delta /(1+\delta)]} .
$$

In addition, a Neimark-Hopf bifurcation occurs when $n^{f}=n_{N H}^{f}(\beta)$ and a flip bifurcation occurs when $n^{f}=n_{F}^{f}(\beta)$.

Note that the local bifurcation curves $n_{N H}^{f}(\beta)$ and $n_{F}^{f}(\beta)$ are independent of the sensitivity coefficients $\gamma, \gamma_{k}$ that scale the sample variances/correlation. As one can easily check, the local stability conditions are exactly the same as in the case (14) of a single risky asset with $\sigma^{2} \equiv \min \left\{\sigma_{1}^{2}, \sigma_{2}^{2}\right\}$. The mathematical and economic insight of this result is fairly intuitive. Given $\rho=0$, the risky assets are only related by the sample correlation coefficient $k_{t}$ which is updated by the chartists. ${ }^{15}$ In the linearized equation for $k_{t}$, the higher order terms disappear and the remaining linear terms will stabilize $k_{t}$ to zero, leading to un-correlated portfolios each with a single risky asset. Hence, the fundamental steady-state of the market with two risky assets is locally stable only if both the fundamental steady-states of the markets with a single risky asset are locally stable. ${ }^{16}$ This remark also holds for the general case of $n$ risky assets. Of course this is only a local result for the particular

\footnotetext{
${ }^{14} \mathrm{~A}$ similar remark holds for the general case of multiple risky assets when the correlation coefficients $\rho_{i j}$ are zero.

${ }^{15} \mathrm{Or}$ by the sample coefficients $k_{i j, t}$ in the case of multiple risky assets.

${ }^{16}$ When one of the two markets is unstable (in the single-risky-asset model) then the steady state of the two-risky-assets model is no longer asymptotically stable, though it may occur in this case that one of the two prices converges to its equilibrium value, see Section 3.3.
} 
case $\rho=0$. In general, due to the presence of $k_{t}$ and $\rho$ in the dynamical system (15), when agents spread their investment among several risky assets, or markets, the resulting time evolution of prices may be quite different from the case where agents specialize in a single asset, or market. In particular, the parameter $\rho$ affects the local stability conditions, while obviously both $\rho$ and $k_{t}$ affect the global dynamics of the system. The impact of the anticipated correlation on the price dynamics, and the role of diversification, compared with the single risky asset case, will be investigated in Section 3.3.

For fixed values of the parameters $R, \mu, \alpha, \delta, a, \sigma^{2}$, Fig. 1 represents, in the space of the parameters $\left(\beta, n^{f}\right), \beta>0,0 \leq n^{f} \leq 1$, the region of local asymptotic stability of the fundamental steady state ${ }^{17}$. Fig. $1 a$ represents the qualitative case $\mu<2 a \sigma^{2} /(R-\alpha)$, where the market maker reaction coefficient $\mu$ is small enough, or the fundamentalist reaction coefficient $(1-\alpha)$ is sufficiently low, i.e. $\alpha$ is sufficiently high. In this case the steady state is locally stable for any fundamentalist fraction $n^{f}$ provided that the chartist extrapolation rate $\beta$ is sufficiently low, while for sufficiently high $\beta$ the steady state becomes unstable, through a Neimark-Hopf bifurcation, when $n^{f}$ falls below the level determined by the Neimark-Hopf curve of equation $n^{f}=n_{N H}^{f}(\beta)$. Numerical evidence suggests that the NeimarkHopf bifurcation is supercritical, in that soon after the crossing of the curve $n^{f}=n_{N H}^{f}(\beta)$ in the space of the parameters, we observe convergence to a stable closed invariant curve in the phase-space. The economic intuition behind the (quasi-)periodic orbits generated from the Hopf bifurcation is that they are due to the lagged response of the trend extrapolated by the chartists to the market price. Fig. 1 b represents the opposite qualitative case $\mu>2 a \sigma^{2} /(R-\alpha)$ (strong market maker or fundamentalist reaction) where the steady state may become unstable also through a Flip-bifurcation, when $n^{f}$ falls above the threshold determined by the Flip curve of equation $n^{f}=n_{F}^{f}(\beta)$. The Flip-bifurcation leads the market price to either jump up and down alternately relative to the fundamental price level or to explode. The economic intuition behind the flip bifurcation comes from the over-reaction of the fundamentalists and market maker. When the market price mean reverts strongly to the fundamental price due to the fundamentalists' behaviour, this over-reaction, together with high speed of the price adjustment of the market maker towards the excess demand (which in this case is dominated by the fundamentalists), pushes the price to over adjust to the fundamental price level, leading it either to fluctuate around the fundamental price or to explode.

Moreover, other dynamic phenomena due to global bifurcations can be observed when parameters are 'far' from the local bifurcation boundaries. Among these, the transformation of a regular attracting closed curve into a torus can sometimes be observed for sufficiently high values of $\delta,{ }^{18}$ that is, when the weight attached to past history in the computation of sample means is sufficiently high; also, the emergence of chaotic dynamics is associated in general with regimes with high $\beta$ and low $\alpha$, which occur when trend followers extrapolate strongly and fundamentalists overreact.

\section{Fig. 1 approximately here}

\subsection{The impact of the correlation on the price dynamics}

The previous section has provided a bifurcation analysis with respect to the key parameters of the model. This analysis has been carried out in the particular case $\rho=0$, where the Jacobian matrix of the dynamical system (15) is merely a double copy of that of the single-risky-asset case (14). The goal of the present section is to perform sensitivity analysis with respect to the correlation structure between

\footnotetext{
${ }^{17}$ In our numerical simulations, the parameters are selected from the stability region corresponding to the qualitative case $2 a \sigma^{2}<\mu(R-\alpha)$ in Fig. 1

${ }^{18}$ This can be observed for instance under the following parameter set: $\alpha=0.75, \gamma=\gamma_{k}=0.5, \beta=\mu=0.5, n^{f}=0.25$, $a=0.05, r=0.05, K=250$ (so that $R=1+r / K=1.0002$ ), $\sigma_{1}^{2}=0.5, \sigma_{2}^{2}=2.5, \rho=0, z_{1}^{s}=z_{2}^{s}=1, \bar{y}_{1}=0.055$, $\bar{y}_{2}=0.155$ which imply $p_{1}^{*}=p_{2}^{*}=150$. For simplicity the parameters $q_{1}$ and $q_{2}$ are set equal to 1 . A torus bifurcates from the existing closed curve when $\delta$ is increased starting from $\delta=0.55$.
} 
the two risky assets. Indeed, anticipated correlation establishes a connection between two separate one-risky-asset markets, via the agents' portfolio allocation. The correlation structure is captured in two different ways within the present model. In the first, the coefficient $\rho$ is the "exogenous" correlation coefficient, which represents the common belief about the equilibrium correlation structure of the two assets, related to the dividend processes. In the second, the model generates endogenously another kind of "perceived" correlation (considered for simplicity only on the part of the chartists) which comes from the historical co-movements of the prices, observed during fluctuations out-of-equilibrium. The impact of this second component is captured by the parameter $\gamma_{k}$, which represents the sensitivity to the sample covariance (see equation (9)). A natural question which arises is whether the perceived correlation and its updating mechanism tends to dampen, or to amplify, the price movements which arise from the interaction of heterogeneous traders. Another closely related question concerns the joint impact of the two components, exogenous and perceived correlations, and which of the two has a dominating effect. It turns out that perceived correlation is not always able to act as a stabilizing force in the presence of endogenous price fluctuations; moreover, one cannot conclude that one of the two components dominates the other one, in general, in that the impact of the correlation structure may change substantially depending on the qualitative behavior of the underlying single-risky-asset markets. In order to study the role of correlation, we express the coefficient $\gamma_{k}$ as a fraction of the sensitivity $\gamma$ to historical volatility by setting $\gamma_{k}=\varphi \gamma, \varphi \geq 0$, with $\gamma_{k}=0$ for $\varphi=0$ and $\gamma_{k}=\gamma$ for $\varphi=1$. The chartist anticipated correlation at time $t$ is thus defined as

$$
\rho_{t}^{(c)}=\frac{\rho \tilde{\sigma}_{1} \tilde{\sigma}_{2} / K+\varphi \gamma k_{t}}{\sqrt{\left(\widetilde{\sigma}_{1}^{2} / K+\gamma v_{1, t}\right)\left(\tilde{\sigma}_{2}^{2} / K+\gamma v_{2, t}\right)}} .
$$

In particular, for $\varphi=1$ the trend followers use the same coefficient $\gamma$ to account for sample variances and covariances. When $\varphi=0$ and in addition $\rho=0$ the demand functions become (for $i=1,2$ ),

$$
z_{i, t}^{f}=\frac{(\alpha-R) p_{i, t}+(1-\alpha) p_{i}^{*}+\bar{y}_{i}}{a q_{i}^{2} \widetilde{\sigma}_{i}^{2} / K} \quad, \quad z_{i, t}^{c}=\frac{(1+\beta-R) p_{i, t}-\beta u_{i, t}+\bar{y}_{i}}{a q_{i}^{2}\left(\widetilde{\sigma}_{i}^{2} / K+\gamma v_{i, t}\right)} .
$$

These are precisely the demand functions of the case $n=1$, obtained by solving a one risky/one riskless asset mean variance optimization problem. Put differently, the case $\varphi=0$ corresponds to a situation where the prices of the two risky assets evolve independently from each other, while in the case $\varphi=1$ investors take into consideration the observed co-movements of prices exactly as they do with their sample volatility: even in the absence of exogenous correlation, i.e. $\rho=0$, this results in interdependent demand functions of the two assets and in prices co-evolving over time.

Let us now examine the joint impact of $\varphi$ and $\rho$ on the dynamics, starting from different qualitative patterns of independent one-risky-asset markets. The following numerical examples are chosen in a way that the two assets have equal fundamental prices. This allows us to represent on the same plane $p, u$ both the phase plot of asset 1 (projection onto the plane $p_{1}, u_{1}$ ) and that of asset 2 (projection onto the plane $p_{2}, u_{2}$ ), using different shading. From a qualitative point of view, we can distinguish between the following cases:-

Case (i): Both and $\varphi$ and $\rho$ have no impact on the long-run price dynamics. This happens in general when the steady states of the two independent one-risky-asset models are locally asymptotically stable, and the parameters are located far from the bifurcation boundaries in the parameter space. The introduction of anticipated correlation into the demand functions has no effect on the price dynamics of the higher dimensional two-risky-asset model, and $\left(p_{1, t}, p_{2, t}\right) \rightarrow\left(p_{1}^{*}, p_{2}^{*}\right)$ independently of $\varphi$ and $\rho$. Intuitively, this is what we would expect.

Case (ii): The exogenous correlation, rather than the perceived correlation, has a major impact on the price dynamics. A typical situation where we get this effect is when one of the two independent markets converges to its fundamental steady state, while the other oscillates on a stable closed curve 
(Fig. 2a). When the markets become interdependent due to positive $\varphi$, then the steady state of the complete model is not locally stable, though in general one of the two assets still converges to its own fundamental steady state (Fig. 2b). This is related to the fact that there exists a stable closed curve belonging to an invariant submanifold of the phase-space and that $\varphi$ has no effect on the eigenvalues evaluated at the steady state. On the contrary, when exogenous correlation $\rho$ is introduced, this peculiar feature is lost and both prices display long-run fluctuations, though the amplitudes of the fluctuations for asset 1 and asset 2 are different (Figs. 2c,d). The higher is $\rho$, the more similar is the amplitude of the fluctuations of the two prices: the stronger link determined by increasing values of $\rho$ causes a spill-over effect from the market with wider fluctuations to the more tranquil market (compare Figs. 2e,f, where $\rho=0.25$, with Figs. 2c,d, where $\rho=0.1$ ). Note that the role of $\varphi$ is irrelevant in practice in this case.

\section{Fig. 2 approximately here}

Case (iii): Both the exogenous and the perceived correlation have a substantial impact on the price dynamics. In particular, while $\rho$ in general has a destabilizing role, the effect of the strength $\gamma_{k}=\varphi \gamma$ of perceived covariance may be stabilizing (case (a) below) or destabilizing (case (b) below), depending on the qualitative nature of the starting situations with two independent portfolios.

Case (a): Fig. 3 represents a case where the role of $\rho$ is destabilizing while that of $\varphi$ is stabilizing. This often occurs when the two independent markets are characterized by fluctuations of similar amplitude on regular closed curves. While $\rho$ destabilizes further the regular closed curves into a torus and then into a more complex attractor (compare Fig. 3a with Fig. 3c and Fig. 3e), the perceived correlation is able to stabilize wide fluctuations in market 2 into convergence to the fundamental price (Fig. 3b) or into fluctuations of reduced amplitude (Fig. 3d), and in particular to change a torus (Fig. 3c) or a strange attractor (Fig. 3e) into a regular closed curve (Figs. 3d and 3f).

Case (b): Fig. 4 represents a case where both $\rho$ and $\varphi$ have a destabilizing impact, in that they cause a transition to irregular fluctuations, revealed by phase plots of increasing complexity. This often occurs when one of the two independent markets fluctuates on a strange attractor, while the other oscillates more regularly (Fig. 4a). In this case we observe a spill-over effect from the market characterized by complex dynamics into the more regular market, and complexity increases in both markets due to both exogenous and perceived correlations (compare Fig. 4a with Fig. 4b, and Fig. 4c with Figs. 4d,e,f).

\section{Fig. 3 approximately here Fig. 4 approximately here}

We have not found cases in which the parameter $\varphi$, rather than $\rho$, has a major impact on the price dynamics. This may indicate that also from the point of view of the global dynamics ${ }^{19}$, the exogenous correlation structure has more impact than does the perceived correlation.

The above numerical experiments lead to very similar results if a negative exogenous correlation (e.g. $\rho=-0.1$ and $\rho=-0.25$ ) is used instead of a positive one, as can be checked numerically. Moreover, the observed phenomena are quite robust with respect to changes of the parameters, and can easily be detected with a range of other parameter constellations.

Summarizing, the two correlations seem to play different roles depending on the stability, or in general the qualitative asset price behavior, of the two separate single-risky-asset markets:

- When both assets in separate markets are stable, both correlation structures have no impact on the price dynamics of the two-risky-asset market;

\footnotetext{
${ }^{19}$ We recall that the parameter $\varphi$ (i.e. $\gamma_{k}$ ) does not affect the local dynamics, while the parameter $\rho$ does.
} 
- When one of the asset prices is stable and the other fluctuates regularly, characterized by a closed curve in the phase plot, a spill-over effect across the markets is mainly caused by the exogenous correlation, rather than perceived correlation, among the two assets;

- When both asset prices fluctuate regularly in the separate markets, a spill-over effect across the markets is mainly caused by the exogenous correlation, while the perceived correlation can stabilize the market price from regular curves to the fundamental price, or from irregular fluctuations, characterized by strange attractors in the phase plots, to regular fluctuations;

- When at least one of the asset prices fluctuate irregularly in the separate markets, a spill-over effect across the markets can be caused by either the exogenous correlation or the perceived correlation, leading to more complicated price dynamics.

\section{Conclusion and further research}

Starting from the single-risky-asset framework proposed by Brock and Hommes (1998) and Chiarella and He $(2001,2003)$, we have developed a multi-asset dynamic model based on the interaction of heterogeneous agents (fundamentalists and trend chasers), who allocate their wealth in each period among several risky assets and a riskless asset, and a market maker, who adjusts the prices of the risky assets depending on the excess demand. The model results, in general, in a high dimensional dynamical system. The basic framework we have used to derive the demands for each risky asset is that of the standard one-period CAPM, the major difference being that agents are allowed to have heterogeneous beliefs about the first and second moments of the distribution of future returns and to update dynamically these beliefs as a function of observed returns. The local stability conditions of the 'fundamental' steady state in the space of parameters have been derived analytically in the particular case of two risky assets and zero "exogenous" correlation. The effect of the correlation structure between the risky assets on the local and global dynamics has been explored by means of numerical simulation - compared with the case where there are two separate single-risky-asset markets. It turns out that in the presence of price fluctuations determined by fundamentalist-chartist interaction, the investors' anticipated correlation is not always able to stabilize the financial market, but it might even act as a further source of complex behavior.

The framework proposed in this paper could be developed in a number of directions.

First the analysis here has focused on a deterministic dynamic model, which could be generalized in order to include the effect of exogenous stochastic factors (e.g. the effect of randomly evolving fundamental prices).

Second, the model here is one with fixed market fractions of fundamentalists and chartists, in the sense that agents are not allowed to "switch" amongst different available strategies on the basis of their realized profits (for instance according to the "adaptive belief system" introduced by Brock and Hommes $(1997,1998))$. The introduction of "switching" mechanisms and time varying proportions would be a necessary and interesting extension of the model.

Third, it would be interesting to analyse how the CAPM relationships are modified in this dynamic framework. For instance, the heterogeneous interacting agent framework, combined with asset diversification and time-varying perception of risk (dynamic updating of variances/covariances) could provide a basis for a theory of time-varying betas, which is widely reported as an empirical fact but poorly explained in the standard CAPM framework.

\section{Acknowledgements}

The authors wish to acknowledge the insightful comments of the referees which helped to clear up some confusing points in the original draft of this paper. The usual caveat applies. C. Chiarella and X.-Z. He acknowledge financial support from the ARC Discovery Project DP0450526. R. Dieci 
acknowledges financial support from MIUR, Italy, within the scope of the national research project "Nonlinear Models in Economics and Finance: Complex Dynamics, Disequilibrium, Strategic Interaction". The authors also gratefully acknowledge financial support from Chuo University (Joint Reseach Project 0382), the Department of Economics and the Japan Ministry of Education, Culture, Sports, Science and Technology (Grand-in-Aid for Scientific Reseach (A) 16203019 and (B) 15330037), and wish to thank the participants to the Third International Conference on Nonlinear Economic Dynamics, Tokyo 2004.

\section{References}

Beja, A., Goldman, M.B., 1980. On the dynamic behavior of prices in disequilibrium. Journal of Finance 35, 235-248.

Böhm, V., Chiarella, C., 2005. Mean variance preferences, expectations formation and the dynamics of random asset prices. Mathematical Finance 15(1), 61-97.

Böhm, V., Wenzelburger, J., 2005. On the performance of efficient portfolios. Journal of Economic Dynamics and Control 29, 721-740.

Brock, W.A., Hommes, C.H., 1997. A rational route to randomness. Econometrica 65(5), 10591095.

Brock, W.A., Hommes, C.H., 1998. Heterogeneous beliefs and routes to chaos in a simple asset pricing model. Journal of Economic Dynamics and Control 22, 1235-1274.

Chiarella, C., Dieci, R., Gardini, L., 2002. Speculative behaviour and complex asset price dynamics: a global analysis. Journal of Economic Behavior and Organization 49, 173-197.

Chiarella, C., Dieci, R., Gardini, L., 2005. The dynamic interaction of speculation and diversification. Applied Mathematical Finance 12(1), 17-52.

Chiarella, C., He, X.-Z., 2001. Heterogeneous beliefs, risk and learning in a simple asset pricing model. Computational Economics 19 (1), 95-132.

Chiarella, C., He, X.-Z., 2003. Heterogeneous beliefs, risk and learning in a simple asset pricing model with a market maker. Macroeconomic Dynamics 7(4), 503-536.

Day, R.H., Huang, W., 1990. Bulls, bears, and market sheep. Journal of Economic Behavior and Organization 14, 299-329.

Fernandez-Rodriguez, F., Garcia-Artiles, M.D., Martin Gonzalez, J.M., 2002. A model of speculative behaviour with a strange attractor. Applied Mathematical Finance 9, 143-161.

Gaunersdorfer, A., 2000. Endogenous fluctuations in a simple asset pricing model with heterogeneous agents. Journal of Economic Dynamics and Control 24, 799-831.

He, X.-Z., 2003. Asset pricing, volatility and market behaviour: a market fraction approach. Quantitative Finance Research Group Working Paper 95, School of Finance and Economics, University of Technology Sydney.

Hommes, C.H., 2001. Financial markets as nonlinear adaptive evolutionary systems. Quantitative Finance 1, 149-167.

Lux, T., 1998. The socio-economic dynamics of speculative markets: interacting agents, chaos and the fat tails of return distributions. Journal of Economic Behavior and Organization 33, 143-165.

Wenzelburger, J., 2004. Learning to predict rationally when beliefs are heterogeneous. Journal of Economic Dynamics and Control 28(10), 2075-2104.

Westerhoff, F.H., 2004. Multiasset market dynamics. Macroeconomic Dynamics 8, 596-616. 


\section{Figure captions}

Figure 1. Local stability region of the 'fundamental' steady state in the space of parameters $\left(\beta, n^{f}\right)$.

Figure 2. Joint effect of $\rho$ and $\varphi$. The case where $\rho$ plays a dominant role and has a destabilizing effect for asset 2. The parameters are the following: $\alpha=0.75, \beta=0.25, \gamma=0.5$ (and $\gamma_{k}=\varphi \gamma$ ), $\delta=\mu=0.5, n^{f}=0.25, a=0.05, r=0.05, K=250$ (so that $R=1+r / K=1.0002$ ), $\sigma_{1}^{2}=0.9$, $\sigma_{2}^{2}=1.6, z_{1}^{s}=z_{2}^{s}=1, \bar{y}_{1}=0.071, \bar{y}_{2}=0.106$ which imply $p_{1}^{*}=p_{2}^{*}=130$ for $\rho=0, p_{1}^{*}=p_{2}^{*}=100$ for $\rho=0.1, p_{1}^{*}=p_{2}^{*}=55$ for $\rho=0.25$. The parameters $q_{1}$ and $q_{2}$ are set equal to 1 for simplicity.

Figure 3. Joint effect of $\rho$ and $\varphi$. The case where both $\rho$ and $\varphi$ have a significant impact on the dynamics of prices: $\rho$ is destabilizing while $\varphi$ is stabilizing. The parameters are the following: $\alpha=0.75, \beta=0.45, \gamma=0.5$ (and $\gamma_{k}=\varphi \gamma$ ) $, \delta=\mu=0.5, n^{f}=0.25, a=0.05, r=0.05, K=250$ $(R=1+r / K=1.0002), \sigma_{1}^{2}=0.9, \sigma_{2}^{2}=1.6, z_{1}^{s}=z_{2}^{s}=1, \bar{y}_{1}=0.071, \bar{y}_{2}=0.106$ which imply $p_{1}^{*}=p_{2}^{*}=130$ for $\rho=0, p_{1}^{*}=p_{2}^{*}=100$ for $\rho=0.1, p_{1}^{*}=p_{2}^{*}=55$ for $\rho=0.25$. The parameters $q_{1}$ and $q_{2}$ are set equal to 1 for simplicity.

Figure 4. Joint effect of $\rho$ and $\varphi$. Case where both $\rho$ and $\varphi$ have a significant, destabilizing impact on the dynamics of prices. The parameters are the following: $\alpha=0.3, \beta=2, \gamma=0.5$ (and $\left.\gamma_{k}=\varphi \gamma\right), \delta=\mu=0.5, n^{f}=0.25, a=0.05, r=0.05, K=250(R=1+r / K=1.0002), \sigma_{1}^{2}=0.9$, $\sigma_{2}^{2}=1.6, z_{1}^{s}=z_{2}^{s}=1, \bar{y}_{1}=0.071, \bar{y}_{2}=0.106$ which imply $p_{1}^{*}=p_{2}^{*}=130$ for $\rho=0, p_{1}^{*}=p_{2}^{*}=100$ for $\rho=0.1, p_{1}^{*}=p_{2}^{*}=55$ for $\rho=0.25$. The parameters $q_{1}$ and $q_{2}$ are set equal to 1 for simplicity. 
Fig. 1
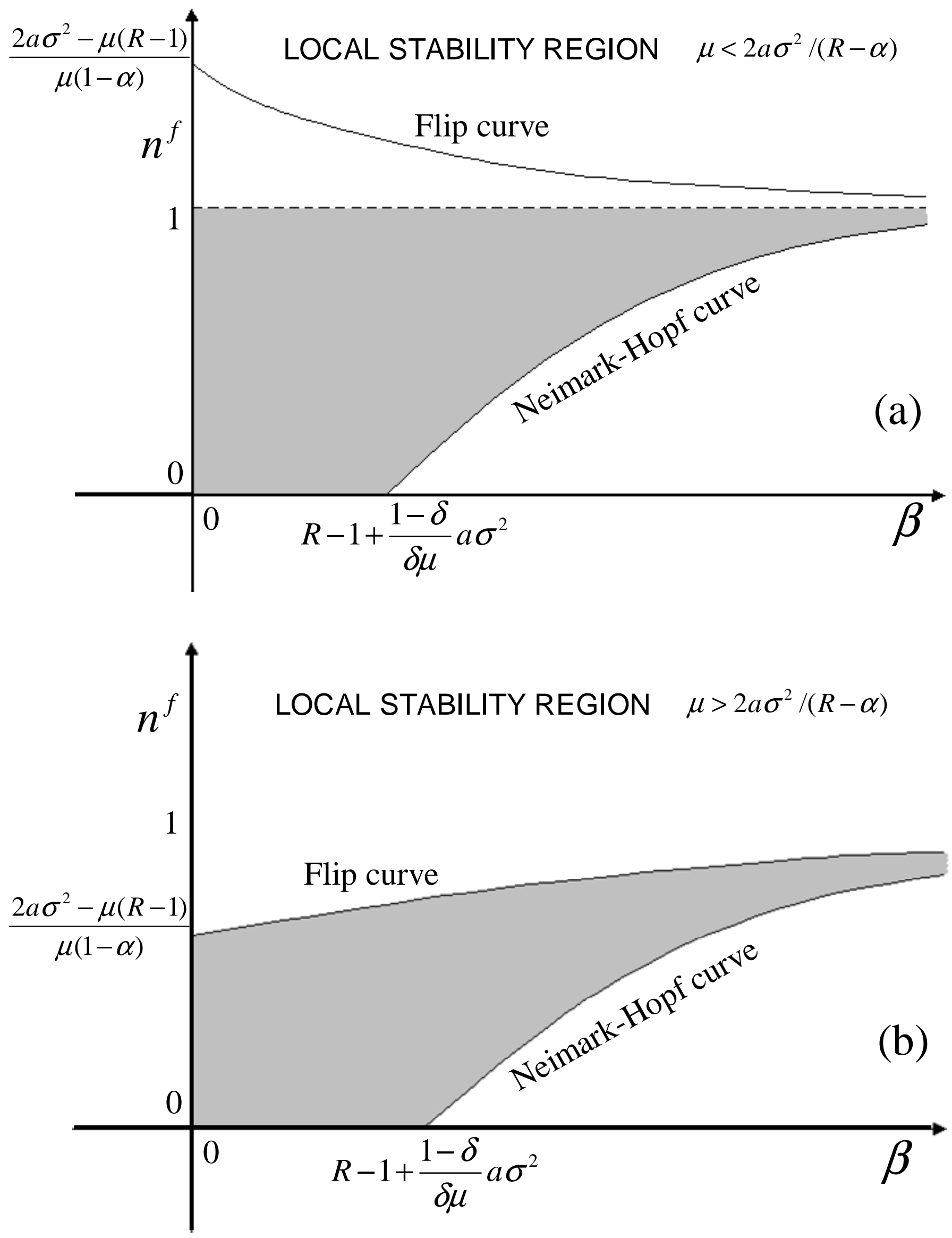
Fig. 2

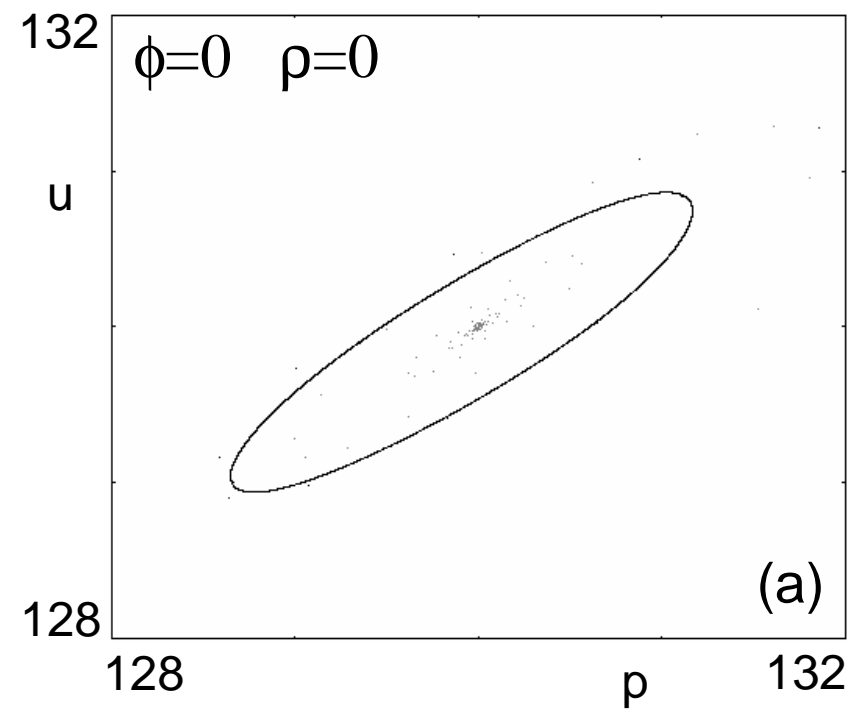

$\phi=1 \quad \rho=0$

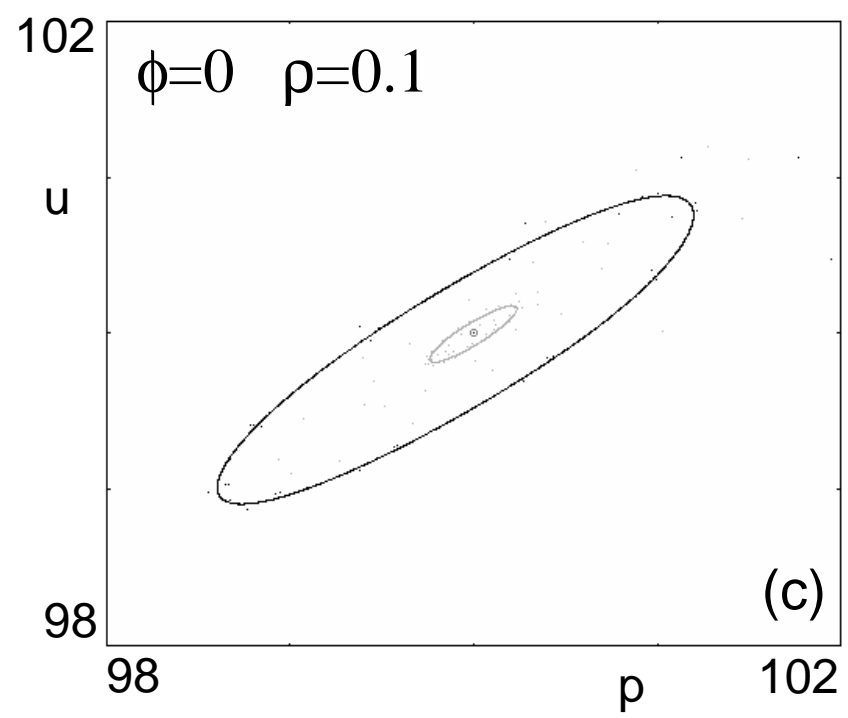

128

u

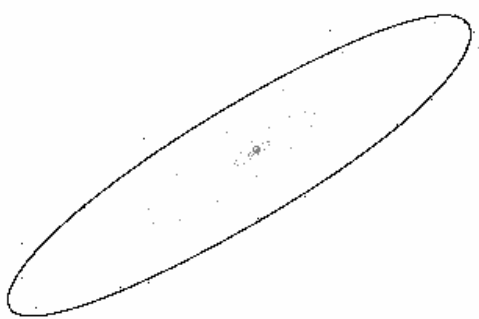

128

132
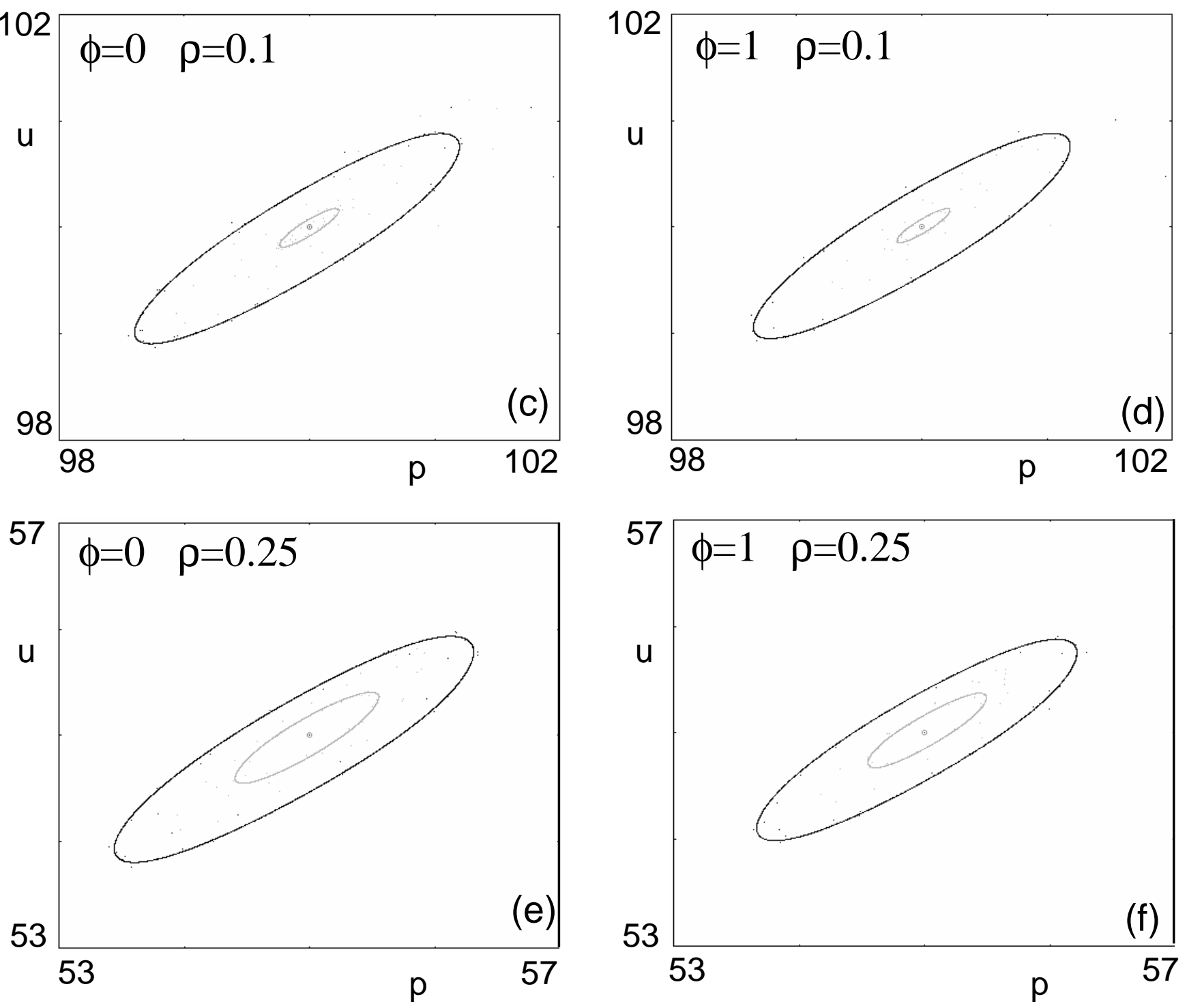
Fig. 3

asset $1\left(p_{1}, u_{1}\right)$

asset $2\left(p_{2}, u_{2}\right)$
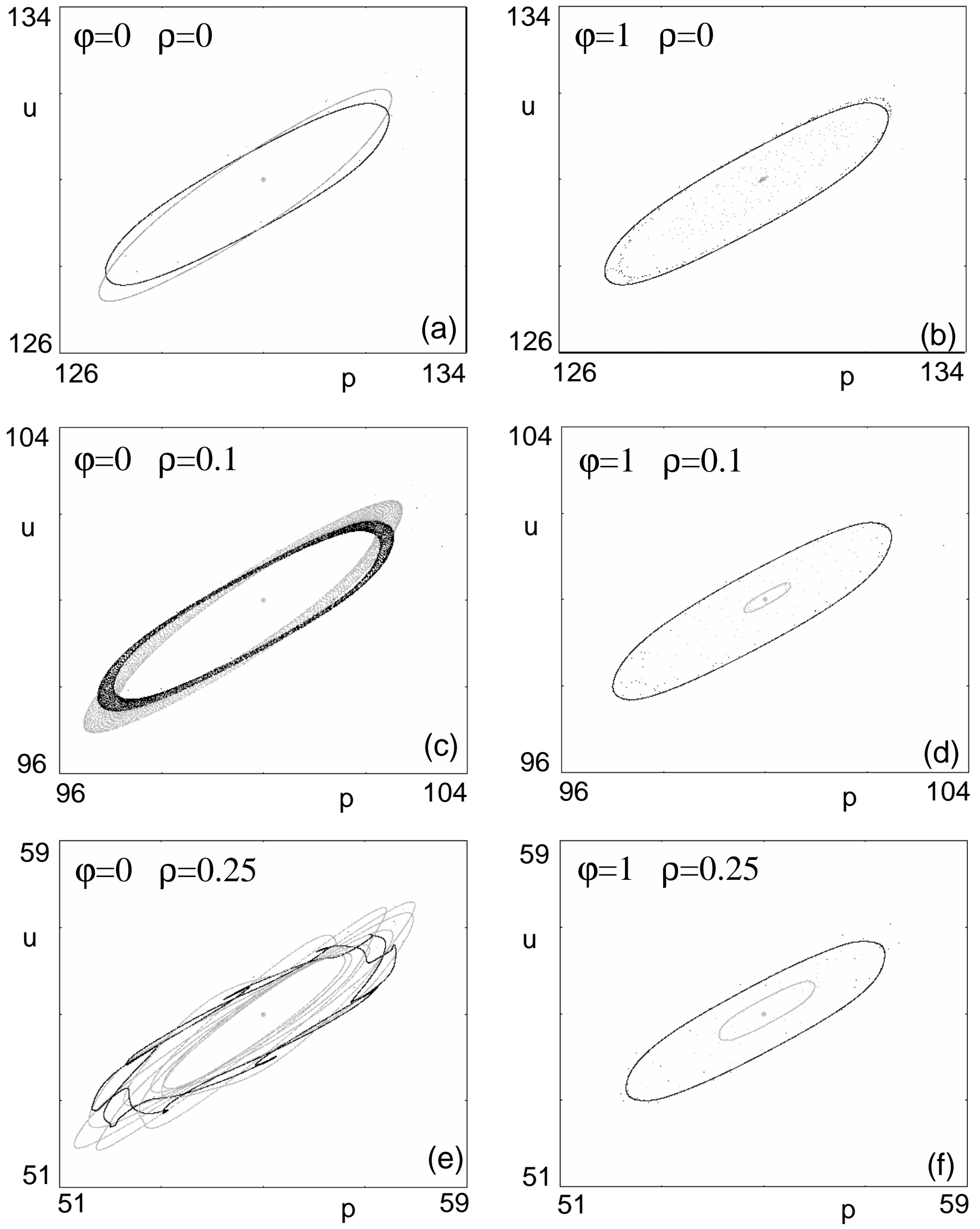
Fig. 4
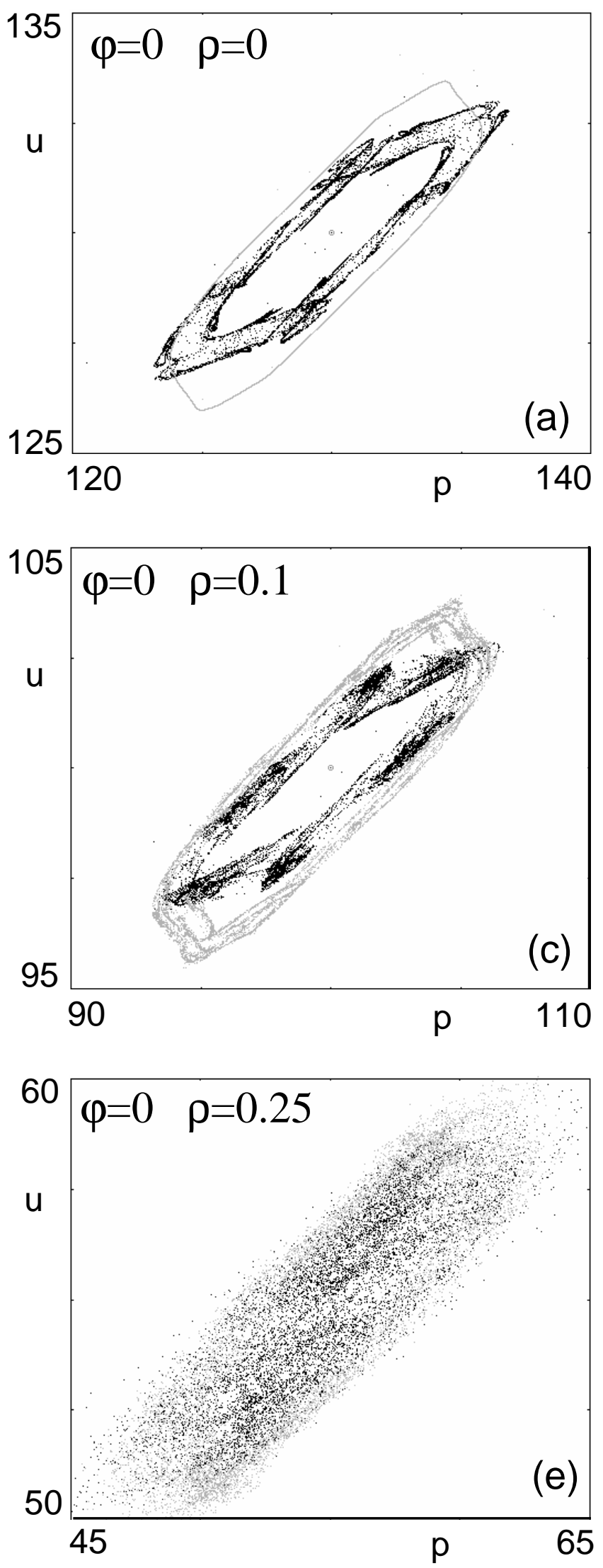

asset $1\left(p_{1}, u_{1}\right)$ asset $2\left(p_{2}, u_{2}\right)$
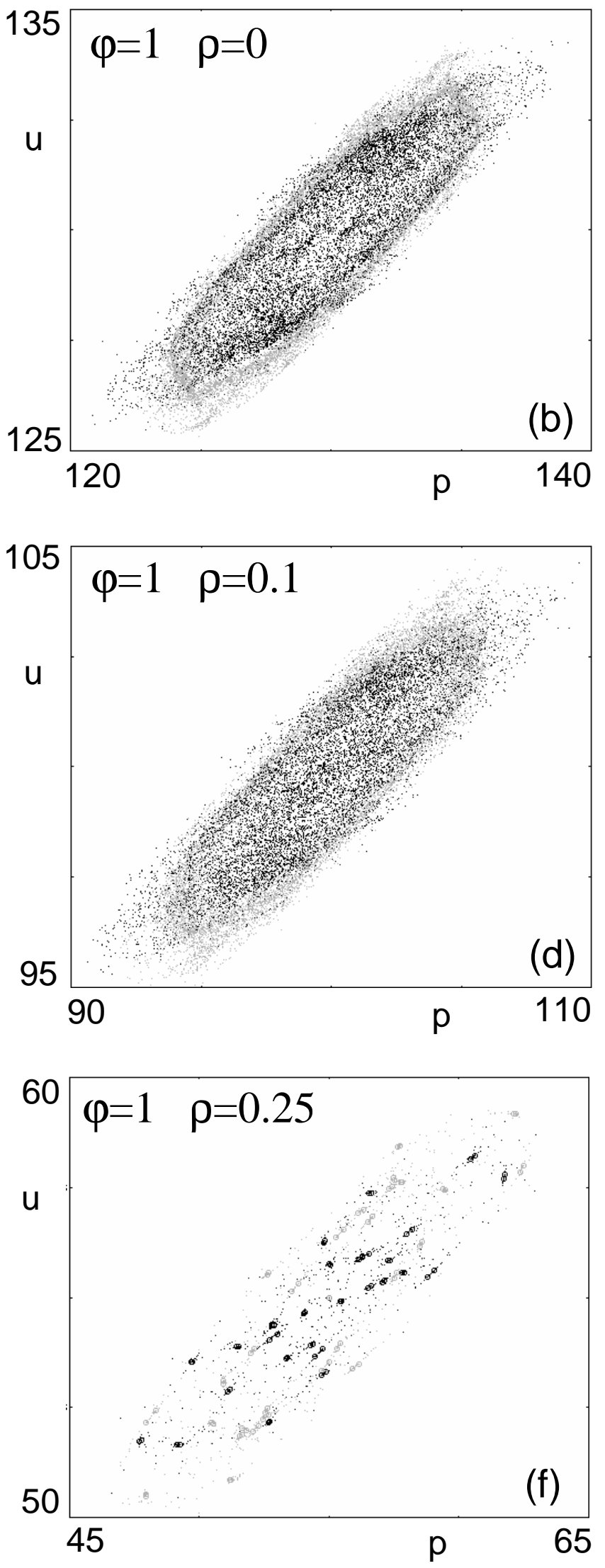